\title{
Y-Axis Voxel Dimension
}

National Cancer Institute

\section{Source}

National Cancer Institute. Y-Axis Voxel Dimension. NCI Thesaurus. Code C156498.

The linear measurement of a voxel along the Y-axis. 